\title{
Risk factors for acquisition of OXA-48-producing Klebsiella pneumonia among contact patients: a multicentre study
}

\author{
D. Hilliquin ${ }^{a, \dagger}$, R. Le Guern ${ }^{b, \dagger}$, V. Thepot Seegers ${ }^{c, d}$, C. Neulier ${ }^{e}$, A. Lomont ${ }^{f}$, \\ V. Marie ${ }^{\text {g, h }}$, C. Legeay ${ }^{\mathrm{a}}$, J. Merrer ${ }^{\mathrm{e}}$, D. Lepelletier ${ }^{\mathrm{i}, \mathrm{j}}$, A.M. Rogues ${ }^{\mathrm{g}, \mathrm{h}}$, \\ B. Grandbastien ${ }^{b}$, J.C. Lucet ${ }^{\mathrm{f}}$, J.R. Zahar ${ }^{\mathrm{k}, \mathrm{l}, *}$
}

a Unité de Prévention et de Lutte contre les Infections Nosocomiales, CHU Angers, Angers, France

${ }^{\mathrm{b}}$ Service de gestion du risque infectieux et des vigilances, CHRU Lille, Lille, France

'Institut de Cancérologie de l'Ouest, Département de Biostatistiques, Pôle de Biométrie, Angers, France

' SFR ICAT, Université d'Angers, Angers, France

e Service de Prévention du Risque Infectieux, CH André Mignot, Versailles, France

${ }^{\mathrm{f}}$ Infection Control Unit, Bichat Claude Bernard hôpital, AP-HP, Paris, France

'Service d'Hygiene Hospitaliere Groupe Hospitalier Pellegrin, Bordeaux, France

${ }^{\mathrm{h}}$ Unité INSERM U1219, Universite de Bordeaux, Bordeaux, France

'Service de bactériologie-hygiène hospitalière, CHU Nantes, Nantes, France

${ }^{j}$ Laboratoire MiHAR, Equipe Emergente, Université de Nantes, Nantes, France

kIAME, UMR 1137, Université Paris 13, Sorbonne Paris Cité, Paris, France

'Département de Microbiologie Clinique, Unité de Contrôle et de Prévention du risque Infectieux, Groupe Hospitalier Paris Seine Saint-Denis, AP-HP, Bobigny, France

\section{A R T I C L I N F O}

\section{Article history:}

Received 8 August 2017

Accepted 29 August 2017

Available online $x x x$

\section{Keywords:}

Carbapenemases

Carbapenemase-producing

Enterobacteriaceae

Risk factors

Screening

OXA-48

Contact patients

\section{S U M M A R Y}

Background: Cohorting carbapenemase-producing Enterobacteriaceae (CPE) carriers during hospitalization limits in-hospital spreading.

Aim: To identify risk factors for CPE acquisition among contacts of an index patient in noncohorted populations.

Methods: A multicentre retrospective matched case-control study was conducted in five hospitals. Each contact patient (case) who acquired Klebsiella pneumoniae (KP)-OXA-48 from an index patient was compared to three contact (controls) with the same index patients matched with hospitalization in the same unit and similar exposure times.

Findings: Fifty-one secondary cases and 131 controls were included. By univariate analysis, exposure time (odds ratio: $1.06 ; 95 \%$ confidence interval: $1.02-1.1 ; P=0.006$ ), concomitant infection at admission $(3.23 ; 1.42-7.35 ; P=0.005)$, antimicrobial therapy within the last month before hospitalization $(2.88 ; 1.34-6.2 ; P=0.007)$, antimicrobial therapy during the exposure time $(5.36 ; 2.28-12.6 ; P<0.001)$, use of at least one invasive procedure $(2.99 ; 1.25-7.15 ; P=0.014)$, number of invasive procedures $(1.52 ; 1.05-2.19$; $P=0.025)$, and geographical proximity $(2.84 ; 1.15-7.00 ; P=0.023)$ were associated with

\footnotetext{
* Corresponding author. Address: CHU Necker Enfants Malades, AP-HP, Université Paris Descartes, Faculté de Médecine, Service Microbiologie-Hygiène, 149 rue de sèvres, Paris 75007, France. Tel.: +33 144494962; fax: +33 144494960.

E-mail address: jean-ralph.zahar@aphp.fr (J.R. Zahar).

Contributed equally to this publication.
} 
CPE acquisition. By multivariate analysis, antimicrobial therapy during the exposure time (odds ratio: 6.36; 95\% confidence interval: $2.46-16.44 ; P<0.001$ ), at least one invasive procedure $(2.92 ; 1.04-8.17 ; P=0.041)$, and geographical proximity $(3.69 ; 1.15-11.86$; $P=0.028$ ) were associated with acquisition.

Conclusion: In this study, geographical proximity, invasive procedure, and antimicrobial therapy during exposure time were significantly associated with KP-OXA-48 acquisition.

(C) 2017 The Healthcare Infection Society. Published by Elsevier Ltd. All rights reserved.

\section{Introduction}

In recent years, several European countries have experienced single-hospital, regional, or inter-regional outbreaks with carbapenemase-producing Enterobacteriaceae (CPE) [1]. This alarming situation warrants prompt preventive measures to limit the spread of highly resistant organisms, and thus reduce a high risk of inadequate antibiotic treatment in cases of infection [2,3].

Experience from Israel demonstrated that quick and strict implementation of preventive measures can contain a countrywide outbreak of carbapenem-resistant Klebsiella pneumoniae [4]. These measures included strict adherence to contact isolation, placement of colonized or infected CPE-positive patients in dedicated wards, and identification and tracking of patient contacts (i.e. patients who were hospitalized in the same unit as a CPE-positive patient and who shared the same caregivers) $[5,6]$.

The 2013 French National Guidelines recommended implementation of a strict isolation policy, including cohorting patients with CPE in a dedicated ward with dedicated healthcare workers (HCWs) and an extensive screening policy of patient contacts [7]. The Parisian experience suggested that strict adherence to recommendations was effective. Indeed, despite an increasing number of CPE index cases, most being linked with a recent stay or hospitalization abroad, the proportion of secondary cases among all CPE cases decreased with cohorting from $69 \%$ to $23 \%(P<0.001)[5]$. However, the number of secondary cases varied significantly depending on the speed and intensity of control measures implemented around the CPE index case. These strict recommendations have two main limitations. First, the costs arising from extensive screening of contact patients and strict contact isolation (sometimes including a loss of beddays), and second, the large number of contact patients lost to follow-up and who are therefore not screened [8].

Being in contact with a CPE-positive patient is the major risk factor associated with CPE acquisition [9]. Several studies have identified other parameters associated with CPE colonization or infection, including previous antimicrobial therapy, prolonged hospital stay, and invasive procedures [10-12]. However, the acquisition of CPE implies the presence of a CPE reservoir, namely another $C P E$-positive patient, and data are lacking regarding risk factors for acquisition in the specific population of contact patients.

The aim of this study was to identify risk factors associated with the acquisition of CPE rectal carriage in a population of contact patients.

\section{Methods}

This multicentre retrospective matched case-control study was conducted in five large French hospitals, including four university hospitals, from August 2012 to December 2015. An index patient was defined as a non-cohorted patient colonized or infected with $K$. pneumoniae carrying the bla $a_{\mathrm{OXA}-48}$ gene (KPOXA-48). These index patients were placed into contact precautions, but without cohorting with dedicated HCWs. We defined contact patients as all the adults (aged $>18$ years) staying in the same unit during the same period as an index patient and sharing the same HCWs. According to French national guidelines, contact patients should be screened weekly using rectal samples [7].

Case patients were defined as contact patients for whom CPE was later identified by the weekly rectal screening. Control patients were defined as contact patients who remained CPE negative during their stay in the hospital, with at least two negative weekly rectal screenings after the end of their exposure.

For each case patient, three control patients were selected on the basis of a similar exposure time. Exposure time was defined as the duration of contact between the index patient and a case patient before being identified as CPE positive. Control patients had at least the same duration of exposure time as the case patients in the same ward. If more than three control patients were available, the patient with the closest exposure time to the case patient was selected, within a maximum range of five days of exposure time between case and control patients.

Data were collected retrospectively in case and control patients by reviewing medical and microbiological records. However, all the patients included in this work were followed prospectively according to the French recommendations [7]. Collected data included demographic characteristics, including age, gender, ward of contact (medical, surgical, or intensive care unit), comorbidities, exposure time, duration of hospitalization in the ward, proximity from the index patient's room, and provenance (hospital, home, nursing home).

Comorbidities were defined as urinary tract abnormalities, neurological disorders, cancer being treated with chemotherapy, immunosuppression, and diabetes mellitus. The Charlson comorbidity index was also recorded [13]. Data related to patients' dependency included: presence of diarrhoea during the exposure time, faecal and urinary incontinence, and the Katz score, evaluating independence in daily activities [14]. Invasive procedures (intravenous central line and/or Foley catheter) during hospitalization were also recorded. Geographical proximity was defined as a room located adjacent to, or in front of, the index patient's room. Sharing of toilets and/or bathroom with the index case was also noted.

Previous antibiotic administration within the previous six months, during the exposure period, and within the week before rectal swabbing was recorded. For statistical analysis, antibiotics were grouped into 12 different classes (penicillins, 
$\beta$-lactams with anti-staphylococcal activity, $\beta$-lactams with anti-anaerobic activity, third-generation cephalosporins, carbapenems, aminoglycosides, fluoroquinolones, macrolides, trimethoprim-sulfamethoxazole, antibiotics with antianaerobic activity, antibiotics with anti-staphylococcal activity, and colistin).

Rectal swabs were used for screening. Swabs were plated on to commercially available CPE selective plates; no broth enrichment was performed. Presumptive colonies were identified using matrix-assisted laser desorption/ionization time-of-flight mass spectrometry (Bruker, Coventry, UK) and in-vitro susceptibility testing was performed by the disc diffusion method in accordance with the guidelines of the Comite de l'Antibiogramme de la Société Française de Microbiologie [15]. Enterobacteriaceae colonies growing on selective plates were also assessed for the presence of the bla $a_{\mathrm{OXA}-48}$ gene throughout the study period. Contacts with positive rectal screening were only classified as cases if the species and type of carbapenemase detected by polymerase chain reaction matched that of the index patient.

\section{Statistical methods}

To evaluate factors potentially associated with CPE acquisition, continuous variables were recorded as mean (standard deviation) and compared using Student's $t$-test; categorical variables were described as proportions and compared by $\chi^{2}$ test (or Fisher's exact test if appropriate). To identify factors associated with CPE, univariate logistic regression analysis was conducted and odds ratio (OR) with $95 \%$ confidence interval $(\mathrm{Cl})$ estimated. The variables yielding $P<0.20$ were introduced in a multivariate conditional logistic regression model. Case and control patients were matched by ward, exposure time, and to the index patient. Thus, our experimental design took into account exposure time as defined above. Despite matching case and control patients on the duration of exposure time, there was a difference between cases and control patients. Consequently, the exposure time was retained in the multivariate model to identify the other potential risk factors after adjustment on this variable. The final model was determined with stepwise variable selection using an automatic procedure based on the Akaike information criterion and was assessed using the Hosmer-Lemeshow goodness-of-fit test. All tests were two-sided and $P<0.05$ was considered significant. To assess the impact of exposure to antimicrobial classes, we compared a treatment with each class in case and control patients using $\chi^{2}$-test or Fisher test as appropriate. The low number of patients in each class, however, precluded antibiotic treatment in the multivariate analysis. Statistical tests were performed using R software 3.2.5 (University of Vienna, Vienna, Austria).

\section{Results}

During the study period, 51 non-cohorted patients carrying or infected with KP-OXA-48 were identified. These patients were hospitalized in different wards. Among them, 23 were readmitted and previously known as carriers, and 28 were identified fortuitously after an average of 17 (range: 3-37) days following hospital admission. Overall, 51 KP-OXA-48positive contacts and 137 control patients were identified.
Table I summarizes demographic data of the 51 cases and their 137 controls. Mean duration of contact in the whole population was 15 days ( \pm 11 days), $52.1 \%$ of our population had received antimicrobial therapy within the last six months, and $52.1 \%$ received antimicrobial therapy during the contact period.

In the univariate analysis, several patient characteristics differed significantly between cases and control patients, including exposure time, antibiotic therapy within the last month and during the exposure time, presence of at least one invasive procedure, number of invasive procedures, concomitant infection, and geographical proximity (Table II).

In the final multivariate regression model (Table II), three independent risk factors for KP-OXA-48 acquisition were identified among contacts: the geographical proximity (adjusted OR: $3.69 ; 95 \% \mathrm{Cl}: 1.15-11.86 ; P=0.028$ ), at least one invasive procedure $(2.92 ; 1.04-8.17 ; P=0.041)$ and antimicrobial therapy during the exposure time $(6.36$; 2.46-16.44; $P<0.001)$.

Case and control patients were matched by their exposure time to an index patient. Despite our matching criteria, the exposure time was significantly higher in cases than in control patients. In the univariate analysis, each additional day of exposure time increased the odds ratio by $3 \%$ (OR: $1.03 ; 95 \% \mathrm{Cl}$ : $1.00-1.07 ; P=0.02$ ). However, the difference was no longer significant in multivariate analysis. However, we kept this variable in the final full model to account for the difference between these matching criteria.

We separately analysed the role of exposure to individual antibiotic classes (Table III), during the exposure time and within the last month before hospitalization. In the univariate analysis, KP-OXA-48 acquisition was associated with a lower exposure to third-generation cephalosporins within the month prior to admission and with higher exposure to $\beta$-lactams with anti-anaerobic activity within the exposure period.

\section{Discussion}

In our large multicentre case-control study, we identified three risk factors associated with KP-OXA-48 acquisition in contacts of a non-cohorted index patient: (i) geographical proximity; (ii) antibiotic therapy during exposure time; and (iii) at least one invasive procedure. In a recent study assessing ICU patients, Schwartz-Neiderman and colleagues suggested that mechanical ventilation, colonization or infection with another multidrug-resistant organism (MDRO), and a contact time of at least three days with an index case were associated with CPE acquisition among contact patients [9]. The risk associated with geographical proximity might be explained by a common healthcare worker and/or by the occurrence recontamination/ transmission through an environmental reservoir.

Several previous studies have reported an association between antibiotic exposure and CPE acquisition. Dautzenberg and colleagues, using a similar study design to ours, identified by multivariate analysis the use of fluoroquinolones within 30 days preceding the index date as the only risk factor for the acquisition of OXA-48-producing Enterobacteriaceae [16]. Many other studies have reported that carbapenem exposure seems to play a major role in the occurrence of CPE acquisition [17-20]. Our results did not specifically identify carbapenems as being associated with acquisition of CPE; however, because 
Table I

Demographic characteristics of the study population

\begin{tabular}{|c|c|c|c|}
\hline Covariate & Total patients $(N=188)$ & Case patients $(N=51)$ & Control patients $(N=137)$ \\
\hline Female sex & $83(44.2 \%)$ & $21(41.2 \%)$ & $62(45.3 \%)$ \\
\hline Age (years) (mean $\pm S D)$ & $68.4 \pm 16.9$ & $69.7 \pm 18.4$ & $68.1 \pm 15.8$ \\
\hline Hospitalization duration (days) (mean \pm SD) & $24.8 \pm 22.7$ & $27.2 \pm 19.4$ & $24 \pm 23.9$ \\
\hline Exposure time (days) (mean $\pm S D$ ) & $14.8 \pm 10.6$ & $17.8 \pm 13$ & $13.7 \pm 9.3$ \\
\hline \multicolumn{4}{|l|}{ Ward of admission } \\
\hline Medical & $151(80.3 \%)$ & $40(78.4 \%)$ & $111(81.0 \%)$ \\
\hline Surgical & $21(11.2 \%)$ & $6(11.8 \%)$ & $15(10.9 \%)$ \\
\hline Intensive care unit & $16(8.5 \%)$ & $5(9.8 \%)$ & $11(8.0 \%)$ \\
\hline \multicolumn{4}{|l|}{ Provenance } \\
\hline Hospital & $110(58.5 \%)$ & $30(58.8 \%)$ & $80(58.4 \%)$ \\
\hline Home & 65 (34.6\%) & $17(33.3 \%)$ & $48(35.0 \%)$ \\
\hline Nursing home & $13(6.9 \%)$ & $4(7.8 \%)$ & $9(6.6 \%)$ \\
\hline \multicolumn{4}{|l|}{ Reason for hospitalization } \\
\hline Infectious & $54(28.7 \%)$ & $20(39.2 \%)$ & $34(24.8 \%)$ \\
\hline Metabolic & $15(8.0 \%)$ & $1(19.6 \%)$ & $14(10.2 \%)$ \\
\hline Neurological disorder & $22(11.7 \%)$ & $7(13.7 \%)$ & $15(10.9 \%)$ \\
\hline Others & $97(51.6 \%)$ & $23(45.1 \%)$ & $74(54.0 \%)$ \\
\hline Charlson score (mean \pm SD) & $4.2 \pm 2.7$ & $4.2 \pm 2.3$ & $4.1 \pm 2.8$ \\
\hline Urinary tract abnormalities & $38(20.3 \%)$ & $8(15.7 \%)$ & $30(22.1 \%)$ \\
\hline Neurological disorders & $49(26.1 \%)$ & $15(29.4 \%)$ & $34(24.8 \%)$ \\
\hline Cancer receiving chemotherapy & $40(21.3 \%)$ & $9(17.6 \%)$ & $31(22.6 \%)$ \\
\hline Immunodepression & $50(26.6 \%)$ & $14(27.5 \%)$ & $36(26.3 \%)$ \\
\hline Diabetes & $61(32.4 \%)$ & $20(39.2 \%)$ & $41(29.9 \%)$ \\
\hline Chronic dialysis & $16(8.5 \%)$ & 7 (13.7\%) & $9(6.6 \%)$ \\
\hline Katz score (mean \pm SD) & $4.7 \pm 4.3$ & $5.5 \pm 4.4$ & $4.4 \pm 4.2$ \\
\hline Urinary incontinence & $44(23.4 \%)$ & $16(31.4 \%)$ & $28(20.6 \%)$ \\
\hline Faecal incontinence & $34(18.1 \%)$ & $11(21.6 \%)$ & $23(16.9 \%)$ \\
\hline Diarrhoea during the exposure period & $24(12.8 \%)$ & $6(11.8 \%)$ & $18(13.2 \%)$ \\
\hline \multicolumn{4}{|l|}{ Antibiotic therapy before hospitalization } \\
\hline $3-6$ months & $98(52.1 \%)$ & $34(66.7 \%)$ & $64(46.7 \%)$ \\
\hline $2-3$ months & $95(50.5 \%)$ & $34(66.7 \%)$ & $64(44.5 \%)$ \\
\hline$\leq 1$ month & $92(48.9 \%)$ & $33(64.7 \%)$ & $59(43.1 \%)$ \\
\hline During the exposure period & $98(52.1 \%)$ & $39(76.5 \%)$ & $59(43.1 \%)$ \\
\hline The week before rectal swabbing & $113(60.1 \%)$ & $34(66.7 \%)$ & $79(57.7 \%)$ \\
\hline Concomitant infection & $112(59.6 \%)$ & $38(74.5 \%)$ & $74(54 \%)$ \\
\hline Presence of invasive procedure & $116(61.7 \%)$ & $39(76.5 \%)$ & $77(56.2 \%)$ \\
\hline Central catheters & 77 (41.0\%) & $26(51.0 \%)$ & $51(37.2 \%)$ \\
\hline Urinary catheters & $47(25.0 \%)$ & $17(33.3 \%)$ & $30(16 \%)$ \\
\hline Nasogastric tube & $28(14.9 \%)$ & $6(11.8 \%)$ & 22 (16\%) \\
\hline Surgery & $9(4.8 \%)$ & $9(17.6 \%)$ & $13(9.5 \%)$ \\
\hline Others & $30(16.0 \%)$ & $9(17.6 \%)$ & $21(15.3 \%)$ \\
\hline Geographical proximity & $23(12.2 \%)$ & $11(21.6 \%)$ & $12(8.8 \%)$ \\
\hline Sharing toilets/shower & $33(17.6 \%)$ & 7 (13.7\%) & $26(19.0 \%)$ \\
\hline
\end{tabular}

of the low number of prescriptions, we were unable to include the different antibiotic classes in our multivariate analysis.

Less than half of the control patients received various antibiotic regimens during the exposure time. Whereas we were unable to assess the role of a specific antibiotic class by the multivariate model, univariate analysis pointed to the possible role of a specific antibiotic class as a risk factor for acquisition. As suggested, third-generation cephalosporins administered within the month prior to admission appeared to be protective, whereas $\beta$-lactams with anti-anaerobic activity during exposure time were associated with a higher risk of CPE colonization. Third-generation cephalosporins have low activity against anaerobic bacteria. Thus, our findings lend support to animal models [21] and clinical studies suggesting that preservation of the anaerobic microbiota may be critical for preventing the acquisition of multidrug-resistant micro-organisms and subsequent infection. It can be hypothesized that a modification of the digestive microbiota by antibiotics, especially those active against anaerobes, compromises resistance to colonization by exogenous strains, with more frequent colonization after potential transmission from HCWs' hands [22]. However, exposure to specific antibiotics in itself is not the only antibiotic-related risk factor; duration and number of treatments might also be significant factors.

Despite being matching criteria, exposure time to the index patient was significantly longer in cases compared with 
Table II

Factors associated by univariate and multivariate analysis with carbapenem-producing Enterobacteriaceae acquisition

\begin{tabular}{|c|c|c|c|c|}
\hline \multirow[t]{2}{*}{ Covariate } & \multicolumn{2}{|c|}{ Univariate analysis } & \multicolumn{2}{|c|}{ Multivariate analysis } \\
\hline & OR $(95 \% \mathrm{Cl})$ & $P$-value & OR $(95 \% \mathrm{Cl})$ & $P$-value \\
\hline Hospitalization duration (days) & $1.01(0.99-1.02)$ & 0.448 & - & - \\
\hline Exposure time (days) & $1.06(1.02-1.10)$ & 0.006 & $1.04(0.99-1.09)$ & 0.112 \\
\hline \multicolumn{5}{|l|}{ Reason for hospitalization } \\
\hline Metabolic vs infectious & $0.07(0.01-0.68)$ & 0.021 & - & - \\
\hline Neuromotor vs infectious & $0.74(0.11-4.86)$ & 0.750 & - & - \\
\hline Others vs infectious & $0.20(0.06-0.71)$ & 0.012 & - & - \\
\hline Katz score & $1.08(0.98-1.19)$ & 0.120 & - & - \\
\hline Urinary incontinence & $1.85(0.83-4.09)$ & 0.131 & $1.95(0.7-5.45)$ & 0.202 \\
\hline Concomitant infection & $3.23(1.42-7.35)$ & 0.005 & - & - \\
\hline Antimicrobial therapy within the last month & $2.88(1.34-6.20)$ & 0.007 & - & - \\
\hline Antimicrobial therapy during the exposure time & $5.36(2.28-12.60)$ & $<0.001$ & $6.36(2.46-16.44)$ & $<0.001$ \\
\hline At least one invasive procedure & $2.99(1.25-7.15)$ & 0.014 & $2.92(1.04-8.17)$ & 0.041 \\
\hline No. of invasive procedures & $1.52(1.05-2.19)$ & 0.025 & - & - \\
\hline Geographical proximity & $2.84(1.15-7.00)$ & 0.023 & $3.69(1.15-11.86)$ & 0.028 \\
\hline
\end{tabular}

$\mathrm{OR}$, odds ratio; $\mathrm{Cl}$, confidence interval.

controls by univariate analysis. We therefore took exposure time into account in the multivariate model. However, exposure time was not associated by multivariate analysis with risk of acquisition, and each additional day of exposure in our study increased the odds ratio of acquisition by $3 \%$. This result suggested that the risk of acquisition was strongly linked to duration of contact, as previously suggested $[9,23]$.

At least one invasive procedure was associated in multivariate analysis with a higher risk of acquisition. Frequent staff contact as a risk factor associated with CPE acquisition is one possible explanation for this observation. Interestingly, we did not find that the Katz score of dependency in daily life activity was predictive of CPE acquisition. This may be because, whereas the Katz index indicates functional status as a measurement of a patient's ability to perform daily activities, it does not specifically take into account the burden of care.

Several case-control studies analysed risk factors for CPE acquisition $[9,20,24-26]$. However, many of them did not take into account the reservoir of $C P E$, i.e. the patients' gastrointestinal carriage, contributing to what is usually described as the 'colonization pressure'. Since colonization pressure is a major risk factor for acquiring MDRO, being able to adjust for colonization pressure is critical for assessing risk factors for CPE acquisition [27-29]. In addition, CPE gastrointestinal tract carriers display a variable risk of dissemination, meaning that assessing CPE acquisition among contact patients around the same index patient helps to obviate the variability associated with the CPE reservoir [30]. Other important determinants of bacterial acquisition are compliance with preventive measures such as hand hygiene or environmental cleaning, specific ward characteristics such as workload or nurse:patient ratio, and the characteristics of the index patients and their contacts.

It should be highlighted that all studies referred to, except one, stem from countries (Italy, Greece, Israel) with different epidemiologic settings (mostly KPC countries) and do not relate to OXA-48-producing CPE $[2-4,6,16]$. Moreover, in contrast to others, our study was conducted in a non-outbreak setting. However, our study has several limitations [16]. First, we assumed that all cases acquired KP-OXA-48 during the exposure time. These patients may already have been colonized prior to their admission or by another index patient during hospitalization. These two situations seem unlikely since France still has a very low prevalence of CPE. High-risk patients with hospital stays abroad within the last year are also expected to be screened at hospital admission in accordance with French recommendations, and contact patients are screened weekly three consecutive times before removing the contact patient status. Second, other factors may play a role in CPE spread, e.g. compliance with preventive measures that was not assessed in our study. Third, we limited our study to KP-OXA-48. Therefore, our results may not be generalizable to settings where other species and/or other carbapenemases are prevalent. In addition, genotypic analysis was not performed to assess the identity of KP-OXA-48 among the clusters under evaluation, and this would confirm a definite link between the index patients and the cases. Finally, although antimicrobial therapy was identified as a risk factor for CPE acquisition, the low number of patients treated by each antimicrobial class prevented us from attributing a possible role to a specific class.

Our results have implications for infection control practice. First, the cohorting of case patients with dedicated HCWs could help to reduce the risk of CPE cross-transmission, compared with contact precautions only. Another major point is that in clinical wards admitting CPE-infected or -colonized patients, antimicrobial therapy, especially $\beta$-lactams with anti-anaerobic activity, was associated with a higher risk of CPE acquisition among contact patients. Therefore, the antimicrobial stewardship team should confirm the usefulness of any antibiotic administered to this specific population. Finally identifying patients with a higher risk of acquisition could help the infection control team to evaluate the individual risk of CPE transmission.

In conclusion, this detailed case-control study identified risk factors for CPE acquisition around an index patient, and emphasized the necessity of combining antimicrobial stewardship and infection control measures for controlling CPE transmission. 
Table III

Antimicrobial therapy

\begin{tabular}{|c|c|c|c|c|c|c|}
\hline Time of therapy & Antibiotic class & $\begin{array}{l}\text { All patients } \\
(N=186)\end{array}$ & $\begin{array}{l}\text { Cases } \\
(N=51)\end{array}$ & $\begin{array}{l}\text { Controls } \\
(N=135)\end{array}$ & OR $(95 \% \mathrm{Cl})$ & $P$-value \\
\hline \multirow{12}{*}{$\begin{array}{l}\text { Antibiotic within the } \\
\text { last month }\end{array}$} & Aminoglycosides & $17(9.1 \%)$ & $2(3.9 \%)$ & $15(11.1 \%)$ & $0.35(0.08-1.60)$ & 0.176 \\
\hline & Carbapenems & $11(5.9 \%)$ & $2(3.9 \%)$ & $9(6.7 \%)$ & $0.64(0.13-3.13)$ & 0.586 \\
\hline & $\beta$-Lactams with anti-staphylococcal activity & $13(7.0 \%)$ & $6(11.8 \%)$ & $7(5.2 \%)$ & $2.31(0.67-7.89)$ & 0.183 \\
\hline & Third-generation cephalosporin & $26(0.1 \%)$ & $2(3.9 \%)$ & $24(17.8 \%)$ & $0.19(0.04-0.83)$ & 0.027 \\
\hline & Fluoroquinolones & $23(12.4 \%)$ & $7(13.7 \%)$ & $16(11.9 \%)$ & $1.17(0.45-3.03)$ & 0.743 \\
\hline & Macrolides & $2(1.1 \%)$ & 0 & $2(1.5 \%)$ & NA & 0.997 \\
\hline & Penicillin A (amoxicillin) & $19(10.2 \%)$ & $6(11.8 \%)$ & $13(9.6 \%)$ & $1.24(0.45-3.45)$ & 0.681 \\
\hline & Antibiotics with anti-anaerobic activity (clindamycin, metronidazole) & $9(4.8 \%)$ & 0 & $9(6.7 \%)$ & NA & 0.997 \\
\hline & $\begin{array}{l}\text { Antibiotics with anti-staphylococcal activity (glycopeptides, } \\
\text { daptomycin, and others) }\end{array}$ & $27(14.5 \%)$ & $7(13.7 \%)$ & $20(14.8 \%)$ & $0.96(0.38-2.44)$ & 0.937 \\
\hline & $\begin{array}{l}\beta \text {-Lactams with anti-anaerobic activity (amoxicillin + clavulanic acid, } \\
\text { piperacillin }+ \text { tazobactam) }\end{array}$ & $42(22.6 \%)$ & $16(31.4 \%)$ & $26(19.3 \%)$ & $1.89(0.91-3.92)$ & 0.086 \\
\hline & Trimethoprim-sulfamethoxazole & $17(9.1 \%)$ & $4(7.8 \%)$ & $13(9.6 \%)$ & $0.79(0.25-2.55)$ & 0.697 \\
\hline & Colistin & $2(1.1 \%)$ & $1(0.02)$ & $1(0.7 \%)$ & NA & 0.997 \\
\hline \multirow{12}{*}{$\begin{array}{l}\text { Antibiotic during contact } \\
\text { period }\end{array}$} & Aminoglycosides & $7(3.8 \%)$ & $2(3.9 \%)$ & $5(3.7 \%)$ & $1.05(0.2-5.58)$ & 0.952 \\
\hline & Carbapenems & $6(3.2 \%)$ & $3(5.9 \%)$ & $3(2.2 \%)$ & $2.71(0.53-13.82)$ & 0.230 \\
\hline & $\beta$-Lactams with anti-staphylococcal activity & $11(5.9 \%)$ & $5(9.8 \%)$ & $6(4.4 \%)$ & $2.41(0.77-7.51)$ & 0.131 \\
\hline & Third-cephalosporin generation & $19(10.2 \%)$ & $2(3.9 \%)$ & $17(12.6 \%)$ & $0.28(0.06-1.27)$ & 0.099 \\
\hline & Fluoroquinolones & $20(10.8 \%)$ & $8(15.7 \%)$ & $12(8.9 \%)$ & $1.88(0.72-4.9)$ & 0.19 \\
\hline & Macrolides & $3(1.6 \%)$ & $1(0.02)$ & $2(1.5 \%)$ & $1.32(0.12-14.75)$ & 0.823 \\
\hline & Penicillin A (amoxicillin) & $7(3.8 \%)$ & $2(3.9 \%)$ & $5(3.7 \%)$ & $1.05(0.2-5.58)$ & 0.952 \\
\hline & Antibiotics with anti-anaerobic activity (clindamycin, metronidazole) & $7(3.8 \%)$ & $2(3.9 \%)$ & $5(3.7 \%)$ & $1.05(0.2-5.58)$ & 0.952 \\
\hline & $\begin{array}{l}\text { Antibiotics with anti-staphylococcal activity (glycopeptides, } \\
\text { daptomycin and others) }\end{array}$ & $21(11.3 \%)$ & $7(13.7 \%)$ & $14(10.4 \%)$ & $1.36(0.52-3.58)$ & 0.532 \\
\hline & $\begin{array}{l}\beta \text {-Lactams with anti-anaerobic activity (amoxicillin + clavulanic acid, } \\
\text { piperacillin }+ \text { tazobactam) }\end{array}$ & $31(16.7 \%)$ & $16(31.4 \%)$ & $15(11.1 \%)$ & $3.6(1.62-7.97)$ & 0.002 \\
\hline & Trimethoprim-sulfamethoxazole & $18(9.7 \%)$ & $7(13.7 \%)$ & $11(8.1 \%)$ & $1.77(0.65-4.84)$ & 0.264 \\
\hline & Colistin & 0 & 0 & 0 & NA & NA \\
\hline
\end{tabular}

OR, odds ratio; $\mathrm{Cl}$, confidence interval; $\mathrm{NA}$, not applicable. 
D. Hilliquin et al. / Journal of Hospital Infection xxx (2017) 1-7

\section{Conflict of interest statement None declared.}

\section{Funding sources \\ None.}

\section{References}

[1] Glasner C, Albiger B, Buist G, Tambić Andrasević A, Canton R, Carmeli Y, et al. Carbapenemase-producing Enterobacteriaceae in Europe: a survey among national experts from 39 countries, February 2013. Euro Surveill 2013;18:20525.

[2] Sypsa V, Psichogiou M, Bouzala G-A, Hadjihannas L, Hatzakis A, Daikos GL. Transmission dynamics of carbapenemase-producing Klebsiella pneumoniae and anticipated impact of infection control strategies in a surgical unit. PLoS One 2012;7:e41068.

[3] Tumbarello M, Trecarichi EM, De Rosa FG, Giannella M, Giacobbe DR, Bassetti M, et al. Infections caused by KPC-producing Klebsiella pneumoniae: differences in therapy and mortality in a multicenter study. J Antimicrob Chemother 2015;70:2133-43.

[4] Schwaber MJ, Lev B, Israeli A, Solter E, Smollan G, Rubinovitch B, et al. Containment of a country-wide outbreak of carbapenemresistant Klebsiella pneumoniae in Israeli hospitals via a nationally implemented intervention. Clin Infect Dis 2011;52:848-55.

[5] Fournier S, Monteil C, Lepainteur M, Richard C, Brun-Buisson C, Jarlier V, et al. Long-term control of carbapenemase-producing Enterobacteriaceae at the scale of a large French multihospital institution: a nine-year experience, France, 2004 to 2012. Euro Surveill 2014;19:20802.

[6] Schwaber MJ, Carmeli Y. An ongoing national intervention to contain the spread of carbapenem-resistant Enterobacteriaceae. Clin Infect Dis 2014;58:697-703.

[7] Lepelletier D, Berthelot P, Lucet J-C, Fournier S, Jarlier V, Grandbastien B. French recommendations for the prevention of 'emerging extensively drug-resistant bacteria' (eXDR) crosstransmission. J Hosp Infect 2015;90:186-95.

[8] Birgand G, Moore LS, Bourigault C, Vella V, Lepelletier D, Holmes AH, et al. Measures to eradicate multidrug-resistant organism outbreaks: how much do they cost? Clin Microbiol Infect 2016;22: 162.e1-9.

[9] Schwartz-Neiderman A, Braun T, Fallach N, Schwartz D, Carmeli Y, Schechner V. Risk factors for carbapenemaseproducing carbapenem-resistant Enterobacteriaceae (CP-CRE) acquisition among contacts of newly diagnosed CP-CRE patients. Infect Control Hosp Epidemiol 2016;37:1219-25.

[10] Ben-David D, Masarwa S, Navon-Venezia S, Mishali H, Fridental I, Rubinovitch B, et al. Carbapenem-resistant Klebsiella pneumoniae in post-acute-care facilities in Israel. Infect Control Hosp Epidemiol 2011;32:845-53.

[11] Borer A, Saidel-Odes L, Eskira S, Nativ R, Riesenberg K, LivshizRiven I, et al. Risk factors for developing clinical infection with carbapenem-resistant Klebsiella pneumoniae in hospital patients initially only colonized with carbapenem-resistant $K$. pneumoniae. Am J Infect Control 2012;40:421-5.

[12] Rivard-Yazigi L, Zahar JR, Le Guillou S, Chalouhi C, Lecuyer H, Bureau C, et al. Risk factors associated with extended-spectrum $\beta$-lactamase-producing Enterobacteriaceae carriage at admission in an infant cohort at a tertiary teaching hospital in France. Am J Infect Control 2013;41:844-5.

[13] Mackenzie CR. A new method of classifying prognostic comorbidity in longitudinal studies: development and validation. 1987. Available at: http://www.aqc.ch/download/HSM_Suppl_ 8_charlson.pdf [last accessed October 2016].

[14] Wallace M, Shelkey M. Katz index of independence in activities of daily living (ADL). 2007. Available at: https://www.
beaconhealthpartners.com/wp-content/uploads/2015/04/Katz Index_01.pdf [last accessed October 2016].

[15] Société Française de Microbiologie. Comité de l'antibiogramme. 2016.

[16] Dautzenberg MJ, Ossewaarde JM, de Greeff SC, Troelstra A, Bonten MJ. Risk factors for the acquisition of OXA-48-producing Enterobacteriaceae in a hospital outbreak setting: a matched case-control study. J Antimicrob Chemother 2016;71:2273-9.

[17] Wang Q, Zhang Y, Yao X, Xian H, Liu Y, Li H, et al. Risk factors and clinical outcomes for carbapenem-resistant Enterobacteriaceae nosocomial infections. Eur J Clin Microbiol Infect Dis 2016;35: 1679-89.

[18] Tașova Y. Risk factors of carbapenem-resistant Klebsiella pneumoniae infection: a serious threat in ICUs. Med Sci Monit 2015;21: 219-24.

[19] Gómez Rueda V, Zuleta Tobón JJ. Risk factors for infection with carbapenem-resistant Klebsiella pneumoniae: a case-case-control study. Colomb Médica 2014;45:54-60.

[20] Ahn JY, Song JE, Kim MH, Choi H, Kim JK, Ann HW, et al. Risk factors for the acquisition of carbapenem-resistant Escherichia coli at a tertiary care center in South Korea: a matched case-control study. Am J Infect Control 2014;42:621-5.

[21] Perez F, Pultz MJ, Endimiani A, Bonomo RA, Donskey CJ. Effect of antibiotic treatment on establishment and elimination of intestinal colonization by KPC-producing Klebsiella pneumoniae in mice. Antimicrob Agents Chemother 2011;55:2585-9.

[22] Ruppé E, Andremont A. Causes, consequences, and perspectives in the variations of intestinal density of colonization of multidrugresistant enterobacteria. Front Microbiol 2013:4.

[23] Vergara-López S, Domínguez MC, Conejo MC, Pascual Á, Rodríguez-Baño J. Lessons from an outbreak of metallo- $\beta$-lactamaseproducing Klebsiella oxytoca in an intensive care unit: the importance of time at risk and combination therapy. J Hosp Infect 2015;89:123-31.

[24] Schwaber MJ, Klarfeld-Lidji S, Navon-Venezia S, Schwartz D, Leavitt A, Carmeli Y. Predictors of carbapenem-resistant Klebsiella pneumoniae acquisition among hospitalized adults and effect of acquisition on mortality. Antimicrob Agents Chemother 2008;52:1028-33.

[25] Mittal G, Gaind R, Kumar D, Kaushik G, Gupta KB, Verma PK, et al. Risk factors for fecal carriage of carbapenemase producing Enterobacteriaceae among intensive care unit patients from a tertiary care center in India. BMC Microbiol 2016;16:138.

[26] Ling ML, Tee YM, Tan SG, Amin IM, How KB, Tan KY, et al. Risk factors for acquisition of carbapenem resistant Enterobacteriaceae in an acute tertiary care hospital in Singapore. Antimicrob Resist Infect Control 2015;4:26.

[27] Austin DJ, Bonten MJ, Weinstein RA, Slaughter S, Anderson RM. Vancomycin-resistant enterococci in intensive-care hospital settings: transmission dynamics, persistence, and the impact of infection control programs. Proc Natl Acad Sci USA 1999;96:6908-13.

[28] Bonten MJM. Colonization pressure: a critical parameter in the epidemiology of antibiotic-resistant bacteria. Crit Care Lond Engl 2012;16:142.

[29] Kaier K, Frank U, Hagist C, Conrad A, Meyer E. The impact of antimicrobial drug consumption and alcohol-based hand rub use on the emergence and spread of extended-spectrum $\beta$-lactamase-producing strains: a time-series analysis. J Antimicrob Chemother 2009;63:609-14.

[30] Lerner A, Adler A, Abu-Hanna J, Cohen Percia S, Kazma Matalon M, Carmeli Y. Spread of KPC-producing carbapenemresistant Enterobacteriaceae: the importance of super-spreaders and rectal KPC concentration. Clin Microbiol Infect 2015;21: 470.e1-7. 